[8] A. AdaM. Journal de Pharmacie et de Chimie, 1881.

[9] KrrNG. Méthodes actuelles d'expertises employées au Laboratoire municipal de Paris. 1921; Dunod, éd.

[10] Hinard. Analyse des laits (Encyclopédie scientifique des aide-mémoire). Gauthier-Villars, éd.

[11] BRuno Röse. Journal d'Industrie laitière, 1888, 157.

[12] Methlł̀re. Journal de Pharmacie et de Chimie, 15 février 1894.

[13] Mernlìne. Journal de Pharmacie et de Chimie, 16 juin 1904.

[14] Metrlère. Bulletin des Sciences pharmacologiques, mai 1908.

[15] Ch. Brioux. Annales de Chimie analytique, 15 octobre 1911, 373.

[16] Bordas et StG. de Raczkowski. C. R. Acad, des Sc., 1902, 134, 1592.

[17] Ch. Marie. C. R. Acad. des Sc, 1899 (2e semestre), 766.

[18] Gutkrn, Pflügers Arch. f. d. ges. Physiol., 95, 107.

[19] Chapman. Journal of Dairy Science, novembre 1928, 11, 6, 429.

[20] Dr N. Gerker et Dr A. Otriker. Traité pratique des essais du lait et produits de laiterie. $8^{\mathrm{e}}$ édition, 1919 ; K. J. Wyss-Erben, éd., Berne.

[21] Wood. The Journal of Biological Chemistry, 1905.

[22] DoRnIc. Journal d'Industrie laitière, 1896, 74.

\title{
CONSIDÉRATIONS PHYSICO-CHIMIQUES SUR LE FROMAGE (1)
}

\author{
par G. KOESTLER
}

Travail de l'Institut de Laiterie et de Microbiologie du Liebefeld.

Directeur-Professeur : $\mathrm{D}^{\mathrm{r}} \mathrm{R}$. BURRI.

Les progrès de nos connaissances sur la nature des gelées nous permettent de plus en plus de comprendre et d'étudier le fromage considéré en tant que système physico-chimique. Le grand bénéfice qui se dégage de ce point de vue pour la pratique provient de ce que de nombreux processus de transformation du fromage sont étroitement liés à cette nature physico-chimique.

Les recherches futures devront s'appuyer sur ces considérations pour étudier la nature physico-chimique du fromage considéré comme milieu microbien et enzymatique des processus de transformation. Logiquement, on devrait arriver par ce moyen à expliquer toute une série de faits concernant le développement des défectuosités du fromage. Le présent travail est une contribution dans cette direction.

On établira tout d'abord quelques données sur la nature physicochimique de la masse du fromage. Dans la deuxième partie, on étudiera comment les recherches physico-chimiques peuvent aider pratiquement à mieux résoudre des problèmes importants. Dans la dernière partie, on exposera quelques-unes des méthodes de recherches qui permettront d'établir certaines propriétés physiques du fromage.

(1) Travail paru dans Landwirtschaftliches Jahrbuch der Schweiz, 1931, p. 421. 


\section{LE LAIT CONSIDÉRÉ COMME SYSTÈME DE TRANSITION PHYSICO-CHIMIQUE DU FROMAGE.}

L'étude de la nature physico-chimique du fromage demande que nous nous occupions d'abord brièvement du système "lait ", ainsi que de ses formes de transition jusqu'au fromage.

Le lait est un système polydispersé, c'est-à-dire que les phases dispersées (graisse, easéine, albumine, lactose, sels, etc.) présentent des particules de différentes grosseurs. D'autre part, nous savons aujourd'hui que le degré de dispersion (grosseur des particules isolées) des phases individuelles peut être d'une grande importance pour le caractère général du système. Plus le degré de division des substances est grand, plus ces substances seront en rapport intime avec le milieu dispersif : l'eau, et plus les forces qui s'exercent à la surface de séparation des particules isolées et de ce milieu dispersif seront grandes.

Subissant l'influence de forces aujourd'hui encore insuffisamment connues (forces de cohésion, d'adhésion, attraction moléculaire, charge électrique, etc.), il existe dans le système lait et dans les conditions physico-chimiques normales, un équilibre quii peut être détruit par des actions mécaniques (processus du barattage), physiques (processus de l'écrémage), chimiques, enzymatiques (processus de caillage), microbiennes (fermentations de toutes sortes). Un des processus les plus importants qui trouble l'équilibre physico-chimique du système lait est celui du caillage. Sans nous arrêter à toutes les théories qui se rapportent au processus de la coagulation par le lab, mentionnons au moins que, dans une des premières phases de ce processus, la substance lab prépare - en dissolvant l'albumine les conditions d'altération de l'équilibre. Les bases théoriques en existent déjà dans la nature physico-chimique particulière du lait, étant donné que certains composants du lait ont déjà, presque normalement, une large tendance à coaguler (phosphate de chaux et caséine).

On admet depuis peu, qu'avant Ia préparation, ces substances sont, avant l'action du lab, dans un état "protégé " (grâce à des colloĩdes protecteurs), que la suppression de cette protection a lieu pendant la première phase de l'action du lab, tandis que dans la deuxième phase, les composants du lait ayant tendance à coaguler, précipitent et en effet, coagulent. La caséine et ses combinaisons calciques sontelles entraînées mécaniquement par le phosphate de chaux? les particules de caséine imbibées d'eau coagulent-elles sous l'influence des changements physico-chimiques? ou des forces particulières de l'action labique participent-elles directement à ce processus de coagulation? On ne le sait! Ce qu'il y a de certain, e'est que, pendant une phase préparatoire, les conditions sont telles qu'il y a altération de l'équilibre du système lait préludant à la coagulation, et que pendant la deuxième phase : la coagulation, la caséine, ainsi que la partie plus 
difficilement soluble du phosphate de chaux, passent presque complètement dans le précipité. Pendant la phase coagulante, il se produit, sous l'influence du lab et avec l'appui de forces particulières à l'ensemble du système lait considéré dans cet état d'altération physico-chimique, une gélification ( $3^{\mathrm{e}}$ phase), c'est-à-dire la transformation en gelée, continue. Ce processus s'accélère sous l'influence d'autres facteurs extérieurs, comme la température, les actions mécaniques, etc., et a comme conséquence que les masses coagulées forment un système aggloméré, dont on peut plus ou moins distinctement reconnaître la structure. Comme toutes les gelées connues, les gelées produites par le lab possèdent certaines forces intérieures propres (force cohésive, d'après A. FoDor) qui provoquent une contraction active de la masse. Toute une série de facteurs internes et externes donnent la mesure de l'importance et de l'intensité de cette contraction. La température joue ici un rôle capital. Puis, les dimensions de la surface supérieure en eontact avec l'extérieur, déterminent pour beaucoup la rapidité de la contraction de la gelée. Le but de la fromagerie est d'augmenter la surface libre de la gelée pendant les premières opérations dans la cuve à fromage (rompage des gelées, fractionnement des grains de caillé, etc.). Grâce aux altérations qui se produisent dans la cuve à fromage, la surface libre de la gelée de fromage est augmentée. L’expression du petit-lait hors des masses de gelée est proportionnelle à cette augmentation de la surface. Ce que l'on considère comme un processus de vieillissement (synérèse) de la gelée dépend de toute une série de facteurs (quantité de lab adsorbée par la gelée, concentration des ions H, température, influences mécaniques, telles que le repos au fond de la chaudière et la préparation mécanique du grain, etc.). Le fromager s'emploie à régler ces influences pour obtenir les qualités propres du fromage arrivé à maturation. Il sait que le travail qui se passe dans la euve à fromage doit suivre dans un rythme déterminé le processus de vieillissement de la gelée qui constitue le fromage (contraction des grains de caillé avec expression du petit-lait). Si le rythme de la fabrication devance l'apparition naturelle de la synérèse, on obtient un grain déchiqueté, petit et peu caractéristique; si, au contraire, il ne la suit pas d'assez près, la masse fromagère est plutôt grossière, il se forme une sorte de peau ferme autour des grains de caillé et le petit-lait s'en exprime moins facilement, ce qui, par la. suite, a une influence défavorable sur la fermentation du fromage. Pour certaines sortes de fromage, il se produit, pendant la fabrication, un "durcissement " du grain que l'on obtient par un chauffage ultérieur à une température élevée et par une longue préparation mécanique du grain du fromage dans la cuve. Outre l'influence microbienne que ces mesures exercent sur le fromage, elles régularisent fortement les propriétés physico-chimiques du grain de fromage, 
pris individuellement. La synérèse en est régularisée jusqu'à un certain point, ce qui agit en même temps sur l'écoulement du petit-lait. Puis, il se produit à la surface du grain de fromage, surtout pendant le chauffage dans la cuve, une zone d'épaississement (peau) dont les propriétés physiques ne seront pas sans influence sur la pâte du fromage. On ne sait rien sur l'origine de cette zone d'épaississement. Il peut s'agir, d'une part, de l'épaississement de la zone superficielle du grain individuel du fromage (formation de la croûte). D'autre part, et en mettant de côté le petit-lait qui entoure le grain du fromage dans la cuve, certaines substances sont déposées ultérieurement, e'est-à-dire pendant la préparation finale dans la cuve à fromage, à la surface des grains de fromage. Nous pensons surtout ici à ces processus que l'on observe fréquemment aux surfaces de séparation des phases dans de simples systèmes physiques. Peut-être les peaux superficielles des grains de fromage qui s'agglomèrent, jouent-elles un rôle dans l'échange général des substances du fromage (diffusion du sel et des produits de dégradation à dispersion élevée). En tout cas, dans le fromage dur, les couches intermédiaires des grains se maintiennent jusqu'à la maturation complète, de telle sorte qu'on arrive toujours à les reconnaître distinctement par des procédés de coloration.

\section{LE FROMAGE EN TANT QUE GELEE.}

Déjà, nos observations concernant l'histoire préalable du fromage nous permettent de considérer la masse fromagère, au point de vue seientifique colloïdal, comme un gel. La notion de gel n'est pas 'assez nette pour qu'elle puisse servir de définition générale. En tout cas, dans la nature, l'état de gel est bien plus répandu qu'on ne l'admet en général. Beaucoup de coagulums qui, à cause de leur faible dégré de dispersion (degré de fractionnement), ne sont pas comptés au nombre des gelées, le sulfate de baryum, par exemple, présentent cependant, dans des eonditions déterminées, des caractères nets de gelées. On a désigné ces gelées sous le nom de coagels (gelées provenant de la coagulation). On emploie souvent les mots gel et gelée comme synonymes, quoiqu'on trouve dans la littérature des opinions qui différencient nettement l'un de l'autre. C'est ainsi que certains chercheurs veulent garder le mot gelée uniquement pour les masses absolument transparentes et élastiques (la gélatine, par exemple). Nous parlerons, par la suite, pour le fromage, d'une gelée, indépendamment du fait que cette désignation est encore discutée scientifiquement, et parce que l'expression gelée est aujourd'hui généralement employée dans la littêrature fromagère.

Les chimistes des colloïdes ont expérimentalement établi une série de gelées anorganiques et organiques, telles que les gelées obtenues du trichlorure de fer, de l'arséniate de manganèse, de l'oxyde de fer, de 
l'oxyde de cuivre, de la silice, du pentoxyde de vanadium, ainsi que de la quinine, de l'amidon, de l'agar, de la gélatine, du savon, etc. Ces gelées, relativement simples au point de vue physique et ehimique, ont servi à expliquer d'une façon plus approfondie la nature seientifique des gelées.

Les propriêtés typiques des gelées sont, d'après R. Zsigmondy :

$1^{\circ}$ L'élasticité et la stabilité de la forme ;

$2^{\circ}$ Une densité faible, et des limites étendues d'élasticité, au contraire des corps rigides et élastiques, tels que l'acier, le verre, ete.;

$3^{\circ}$ La possibilité d'une plus grande déformation, sans se rompre, au contraire des masses plastiques, telles que la poix, les bouillies, etc.;

$4^{\circ}$ Elles se composent généralement de deux parties : une solide et une liquide ;

$5^{0}$ Proportionnalité entre le degré de compacité et la teneur en colloïdes solides;

$6^{\circ}$ Une faible quantité de substances solides suffit à donner aux gelées leurs propriétés typiques.

On a édifié de nombreuses théories sur la structure des gelées les mieux connues. D'un côté, les théories d'après lesquelles on admet pour tous les corps un état cristallin, de l'autre, celles qui admettent un état amorphe. Dans tous les eas, J. M. Katz et d'autres ont réussi à établir dans des gelées typiques des cristaux en tant qu'éléments fondamentaux de structure. C'est ainsi que dans le caoutchouc, grâce à la radiographie, ces cristaux furent observés. Lors d'un fort étirement de la masse, ils s'orientaient d'une manière très particulière, de telle façon que le plus grand axe des cristaux était parallèle au sens de l'étirement. G. WIEgner a établi dans des gelées de pentoxyde de vanadium - comme d'ailleurs dans une gelée organique typique, la gelée de fibrine - la présence d'ultra-microns en forme de bâtonnets. Le passage de l'état liquide (sol) à l'état de gel est caractérisé dans le pentoxyde de vanadium par le parallélisme des petits cristaux de la phase dispersée, c'est-à-dire du pentoxyde de vanadium. On sait qu'une gélatinisation peut être provoquée ou accélérée dans le "sol " de fibrine (défibrinisation du sérum sanguin) par une action purement mécanique (agitation ou battage de la solution de fibrine). Si ces exemples justifient l'hypothèse d'une structure cristalline des gelées simples, ne contenant qu'une individualité chimique, on n'a pu encore démontrer la structure cristalline des gelées de compositions plus compliquées. On s'est jusqu'à présent contenté d'étudier leur structure grossière. La structure en cellules hexagonales de Bütschli est caractérisée par la disposition des parties composantes, sous la forme d'écume analogue à des rayons d'abeilles. $\mathrm{O}$. Bütschli trouve que cette forme de structure est la plus répandue, et il en conclut que la 
formation des gelées repose sur la séparation de deux liquides auparavant mélangés. D'après Wo. OstwaLD, la gelée est un système de deux phases liquides entre lesquelles se trouve une surface-limite: pourvue d'une certaine tension. D'après E. NÄGELI, les éléments de structure les plus grossiers des gelées dérivent des éléments les plus fins; ces derniers sont dénommés "micelles ». R. Zsigmondy, lors de ses recherches sur la gélatine au stade de la formation de gelée, observe d'innombrables ultra-microns qui se précipitent ensemble en flocons (microscopiquement et submicroscopiquement). Au début, les ultra-microns étaient encore en mouvement (le mouvement brownien). Ce mouvement cessait peu à peu et le floconnement acquérait une certaine solidité interne. Ceci mène, d'après R. ZsIGMONDY, à la structure "granuleuse " des gelées.

Cet état granuleux des gelées devrait, au point de vue de la structure grossière, entrer aussi en considération, au premier chef, pour les gelées de fromage. En tout cas, la masse fromagère peut être considérée - d'après l'état actuel de la science - comme une véritable gelée où les phases dispersées (complexe Paracaséinate-Phosphate de chaux) apparaîtraient sous forme d'assemblage de micelles entrelacés (réunion de plusieurs micelles en plus gros assemblages). La graisse est alors enfermée mécaniquement à l'état liquide. L'eau et les parties liquides existent dans la masse de la gelée, soit intermicellaires ou intramicellaires. On admet, en outre, que l'enzyme lab est fixé par adsorption à la surface des micelles et de leurs éléments plus fins. Le processus de synérèse (vieillissement de la gelée) détermine la quantité de 'substances liquides qui, dans des conditions extérieures constantes (pression, température, etc.), sont retenues par la gelée, ce qui conduit à l'emprisonnement d'une quantité de substances fermentescibles strictement limitée par la fabrication.

\section{THÉORIE DE LA FORMATION DE LA GELÉE.}

Les processus intimes quí mènent à la formation de la gelée ne nous sont pas encore connus. A. FoDor résume ainsi les opinions connues jusqu'ici :

" Par condensation, des assemblages moléculaires très chargés de solvants apparaissent dans le sol gélatinisant; les assemblages sont soumis à l'action de forces de cohésion élevées qui existent entre les particules du sol, de sorte que tout le système conserve une structure homogène, même après gélatinisation complète. "

A propos de la formation de la gelée, A. FoDor distingue deux cas différents :

a) Transformation immédiate des particules du sol en gelée, faisant ainsi obstacle à une organisation structurale en grille et à la cristallisation. 
b) Le sol est gélatinisé par coagulation.

Mais il faut admettre que dans les deux cas, la structure cristalline ne se forme qu'ultérieurement, c'est-à-dire lors du vieillissement de la gelée.

Autant que nos propres expériences nous l'ont montré, le caillage du lait par le lab, lors de la gélatinisation, est précédé d'une coagulation (diminution des sub-microns de la caséine et augmentation correspondante de la grandeur des particules au moment de la gélatinisation).

Si nous voulons envisager au point de vue critique les différentes théories de la formation des gelées, sur leur application et leurs rapports particuliers avec la formation du fromage, on pourrait dire brièvement ce qui suit :

On a considéré pendant un temps la structure en cellules déjà rappelée comme la forme dominante de la structure des gelées. Mais d'après A. FoDor, elle n'apparaît que dans les sols concentrés, la phase dissoute se séparant alors en dispersion grossière et le milieu de dispersion ne formant finalement que des couches relativement minces entre les particules de la solution (voir aussi J. R. Katz).

Dans beaucoup de cas, la phase dispersée, liquide, s'écoule en gouttelettes et. il se produit un réseau et une structure en forme d'écume. D'après les recherches de Zsigmondy et Bachmann, les examens optiques des gelées de l'acide silicique, de la gélatine, de l'agar, des savons, etc. (ainsi qu'il en a été dit plus haut), démontreraient une structure granuleuse. On admet alors que dans ces gelées, les particules (micelles de Naegli, amicrons et submicrons de la phase dispersée) sont séparées les unes des autres par des intervalles capillaires, si bien que l'ensemble ressemble à une éponge avec des espaces capillaires grossiers, fins et très fins. Les espaces capillaires sont, en même temps, le siège d'énergies importantes et actives, paŕmi lesquelles l'attraction capillaire (force superficielle qui conditionne par son énergie extraordinaire la retenue des substances liquides).

L'hypothèse que des espaces capillaires de différentes sortes se trouvent dans la gelée, fait comprendre que l'eau s'y trouve retenue par des forces très différentes. Tandis que l'eau s'échappe des espaces intermédiaires larges dans les conditions de pression et de température ordinaires, il faut des forces importantes pour expulser les dernières traces d'eau.

La propriété de la gelée, de retenir une quantité d'eau correspondante à la grandeur de sa surface intérieure, ne change qu'avec le temps, avec le vieillissement de la gelée ou sous certaines influences physico-chimiques. Nous en avons déjà parlé et nous avons dit que dans la gelée fraîche de fromage, cette tendance d'expulsion du petitlait à la surface libre apparaît déjà tôt après sa formation. 
On peut admettre que, sous l'action d'une transformation des substances de soutién de la gelée, les espaces capillaires les plus fins disparaissent peu à peu ou s'élargissent, de sorte qu'une partie de l'eau peut s'échapper ou même être expulsée par les pressions qui s'exercent.

L'analyse a démontré depuis longtemps que les gelées retiennent l'eau avec une intensité différente, le premier séchage des gelées dans les conditions de température, de pression et d'humidité ordinaires. s'effectuant facilement. Plus la gelée est sèche, plus on doit prendre de mesures particulières pour faire sortir encore d'autres quantités d'eau de la gelée. La dernière humidité peut être retenue de telle façon dans une gelée, qu'on soit tenté de penšer à une combinaison chimique.

A. Fodor différencie dans les gelées les diverses combinaisons aqueuses suivantes :

10 Eau statique (moléculairement fixée);

20 Eau d'adsorption : a) dynamique, c'est-à-dire faiblement retenụe; $b$ ) fixée par adsorption, c'est-à-dire assez fortement retenue ;

$3^{0}$ Eau micellaire (eau contenue dans les capillaires des plus grandes particules de substances);

$4^{\circ}$ Eau d'imbibition (eau contenue mécaniquement dans les plus grands vides des gelées).

\section{GONFLEMENT.}

Un état particulier des gélatines est celui du gonflement. Celui-ci est presque toujours propre aux corps imbibés d'eau, et atteint son maximum dans la gelée. Le gonflement consiste en ce que le liquide est admis à l'intérieur du corps qui va s'imbiber, et cela se produit dans les règles suivantes:

10 Augmentation de volume;

$2^{\circ}$ Diminution de densité ;

3̧० Augmentation d'élasticité.

Ce gonflement peut sembler illimité. Dans ce cas, par suite de la continuelle fixation d'eau, il se produit un mélange parfait du solvant et du corps dissous (colloïde), si bien que la solution apparaît liquide ou demi-liquide. Mais cela ne veut pas dire que de cette façon se produise une véritable solution (division dispersée moléculaire de la phase dissoute) ; la phase divisée peut très bien continuer à demeurer dans un état colloïdal reconnaissable à l'ultra-microscope.

Le gonflement se poursuit dans des conditions déterminées et scientifiquement observées. Il se fait par paliers qui se succèdent plus ou moins rapidement. 
Au premier palier, l'eau est rapidement absorbée, puis plus lentement au second. Ce palier est suivi d'un troisième, où la fixation de l'eau est très faible, et d'un dernier, où le gonflement atteint lentement son maximum. Si nous faisons entrer cette fixation de l'eau dans un système de coordonnées où les temps sont en abcisses et les quantités d'eau absorbées, en ordonnées, nous obtenons la courbe caractéristique du processus de gonflement établie par VAN BEMMELEN, J. R. Katz et autres. Lors du dégonflement, le retrait du liquide s'effectue en sens contraire, tout au plus avec une légère différence.

VAN BEMmelen a étudié le gonflement et le dégonflement dans la gelée d'acide silicique, en conservant dans des exsiccateurs la substance à gonfler et dégonfler à différentes tensions de vapeur (sur l'acide sulfurique à différentes concentrations), et il détermina constamment, après des intervalles de temps dissemblables, la teneur aqueuse de la gelée.

L'influence des différents sels sur le gonflement a été examinée par F. HoFMEISTER. Les règles suivantes en traduisent les résultats (essais avec gélatine) :

10 Ce qui élève l'élasticité de la gelée diminue sa faculté de gonfler ;

$2^{\circ}$ Les sels qui empêchent le gonflement sont, selon un ordre décroissant : sulfate, tartrate, citrate, acétate de $\mathrm{Na}$;

$3^{\circ}$ Les sels qui activent le gonflement sont, selon un ordre croissant : $\mathrm{NaCl}, \mathrm{KCl}, \mathrm{NH}^{4} \mathrm{Cl}, \mathrm{NaClO}^{3}, \mathrm{NaNO}^{3}, \mathrm{NaBr} \ldots$

Pour la clarté de ce qui va suivre, mentionnons que le sel de cuisine $(\mathrm{NaCl})$ est à la tête des sels qui activent le gonflement des gelées.

Les résultats suivants des expériences faites par R. Zsiamondy sur la gelée de gélatine sont aussi intéressants.

La gélatine absorbe l'eau et le sel des solutions salines, et l'absorption d'eau augmente avec la concentration saline jusqu'à un maximum, puis elle diminue. Par contre, l'absorption de sel augmente à peu près proportionnellement avec la concentration saline dúliquide extérieur. Lors de plus fortes concentrations salines du liquide extérieur, elle a lieu jusqu'à ce que la concentration saline de la gélatine soit pareille à celle de la solution saline environnante.

Des règles semblables s'appliquent pour la gelée de fromage fraîche. Des recherches approfondies en étudieront les écarts provoqués par les altérations croissantes du fromage.

\section{PROBLEMMES PRATIQUES IMPORTANTS.}

Nous allons essayer, en nous appuyant sur les données scientifiques énoncées ci-dessus, de jeter un coup d'œil d'ensemble sur des problèmes qui relèvent du point de vue pratique. 
Comme il a déjà été dit plus haut, les examens de nature strictement ehimico-colloïdale sur la formation et la nature des gelées du lab manquent jusqu'ici. A ce propos, nous posons la question de savoir si une coagulation par électrolytes précède la formation de la gelée dans le processus du caillage. C'est ici que des examens microscopiques et ultra-microscopiques, avec emploi d'une technique d'éclairage variée et appropriée, peuvent donner des conclusions utiles. Des examens physico-chimiques faits sur des gelées de fromage récentes et continués sur de plus anciennes, peuvent aussi nous donner des conclusions sur les différences de propriétés structurales des gelées de fromage (et vraisemblablement plus que l'on pourrait en attendre de l'étude uniquement chimique du problème).

A ce propos, on ne doit pas négliger de considérer l'influence des processus microbiens qui ont eu lieu antérieurement dans le lait. Les altérations physico-chimiques de la gelée pendant la fabrication nous sont encore peu connues. Les processus qui ont leur siège à la surface des grains de fromage isolés sont ici d'un grand intérêt. Le grain de fromage isolé, siège de l'activité des microorganismes et des enzymes, doit être étudié de plus près encore. Nous ne savons rien de certain sur les processus plus subtils dans le grain de fromage pendant la fabrication avec des matières premières présentant des propriétés en dehors de la moyenne (lait trop mûr, à coagulation paresseuse, alcalin ou anormal autrement.) De même, pour l'influence d'une présure à forte ou faible acidité. L'influence aussi de diverses données de la fabrication (courbe de température pendant la préparation du gel, addition d'eau au lait de la cuve, etc.) sur la qualité du fromage en maturation, n'est pas encore étudiée scientifiquement. La découverte d'un procédé d'examen sûr - procédé qui permettrait d'exprimer par des chiffres la qualité de la gelée produite avec la présure, comme aussi d'établir, indépendamment du procédé empirique, l'état du grain de fromage que l'on sait devoir atteindre pour obtenir par compression la qualité normale du fromage - aurait une grande valeur pratique.

De même, les connaissances exactes sur l'action d'une série de mesures pratiques de la fabrication, comme : le "laisser reposer ", le brassage final, etc., de la masse de fromage, manquent encore. Un processus à observer également est la fusion des grains isolés en une masse plus ou moins compacte. La question sera particulièrement importante de savoir quelles sont les altérations physico-chimiques que la gelée de fromage subit sous l'action des fermentations, de la salaison, du chauffage (local de fermentation), etc. On consacrera aussi une étude aux processus de diffusion quand le fromage est frais ou vieux. De même que les influences des transformations chimiques dans le fromage, la dégradation de la paracaséine doit avoir un effet 
sur les propriétés physico-chimiques du fromage; au cours des modifications chimiques de la substance du fromage, se produisent sûrement des transformations qui affectent aussi la structure de la gelée de fromage. Puis, les processus de la formation des trous, qui semblent bien expliqués dans leur partie chimico-biologique, sont, par contre, encore bien obseurs au point de vue physico-chimique. La diffusion des gaz, leur rassemblement en des lieux déterminés du fromage, les conditions de formation des trous normaux et anormaux (forme, grandeur et bordure), toutes ces questions sont de la plus grande importance pratique. On êtudierait aussi l'influence de la température dans la cave de fermentation, ses rapports avec la formation de la croûte et les modifications intérieures du fromage.

Après la fermentation principale, se produisent des modifications qui se révèlent à l'extérieur seulement, par diverses altérations de forme, et à l'intérieur par une altération nette de la pâte de fromage. Puis viennent les modifications dans la cave à fromage, pendant la maturation, en magasin, où l'influence principale du sel, ainsi que celle de la dégradation plus profonde des albumines, sont intéressantes. Là, les considérations purement chimiques ne nous aideront pas, aussi longtemps qu'une méthode n'aura pas été trouvée qui nous permettra de saisir quantitativement et à chaque stade de la maturation les produits isolés de transformation. On pourra peut-être, par l'emploi d'une méthode d'examen physique appropriée, venir à bout de cette immense difficulté. Les altérations du fromage pendant la maturation en magasin sont encore peu connues, et elles jouent cependant un rôle prédominant dans l'aspect et le goût définitif du fromage arrivé à maturation. Pour ce qui est du goût, un certain rapport entre le sel de cuisine et les substances qui déterminent le gô̂t y joue un rôle. La consistance du fromage d'Emmenthal, arrivé à maturation; est un autre point qui intéresse au plus haut degré le chimicophysicien. Cette consistance est dépendante des rapports de structure, des produits de transformation de la masse du fromage, du sel de cuisine et d'autres facteurs physico-chimiques qui, dans le fromage en maturation, sont entraînés dans un perpétuel échange. Les questions pratiques, comme le magasinage du fromage à différentes températures, ont souvent été traitées; par contre, on manque d'expériences qui pourraient nous renseigner sur les altérations plus subtiles du fromage pendant ce magasinage. Il en est de même pour l'influence extraordinaire des températures basses sur les propriétés et la conservation ultérieure du fromage (congélation du fromage).

En cherchant à donner un aperçu des problèmes qui, dans la pratique fromagère, s'imposent au chimico-physicien, nous le faisons à dessein sous forme d'une sorte de programme de travail exposant. les problèmes à résoudre. Nous n'avons pas la prétention d'être 
complet et nous savons bien que e'est peu d'aroir seulement indiqué ces problèmes. Nous savons aussi que toute une série de ces problèmes sont d'une grande difficulté scientifigue. Il ne peut done s'agir que d'un programme à larges vues. Malgré cela, tout essai qui s'occupera sérieusement de résoudre un de ces nombreux problèmes au point de vue de la pratigue de la fromagerie, sera le bienvenu.

(A suivre.)

\title{
ÉTUDE COMPARATIVE DES PRINCIPALES CONSTANTES UTILISÉES POUR LA DÉTERMINATION DU MOUILLAGE DU LAIT (1)
}

\author{
par J. CERE
}

Ingénieur de l'Institut agricole et diplômé de l'Ecole de Laiterie de la Faculté des Sciences de Nancy, Ancien assistant de l'Etablissement fédéral d'Industrie laitière et de Bactériologie du Liebefeld, Berne.

INTRODUCTION.

Définition. - En chimie laitière, on appelle "constantes" des formules chiffrées résultant d'analyses multiples, qu'on a eherché à rendre constantes, c'est-à-dire stables.

Or, nous dit DUCLaUX : " Rien n'est moins constant que les constantes du lait. " Cet aphorisme n'est nullement une boutade, car ces constantes n'ont en effet pas toujours la même valeur. Elles varient selon le procédé qui leur a donné naissance. C'est pourquoi DucLaux a pu dire également : "Il n'y a pas un lait, il y a des laits. "

Il est évident que la composition chimique du lait varie par suite de causes nombreuses. Certains éléments constitutifs de ce liquide s'y rencontrent en quantité dont les proportions peuvent osciller entre 1 et $50 \%$. Cet état de choses rend la détermination des fraudes délicate et aléatoire, si l'on s'en tient à l'analyse quantitative des éléments qui entrent dans la composition du lait.

Pour obvier à ces inconvénients, les chimistes ont eu recours à d'autres procédés physiques ou chimiques indépendants des variations quantitatives. Ces procédés, traduits en formules chiffrées, s'appellent, comme nous l'avons dit : "Constantes du lait ".

Suivant leur origine, nous les elassons en deux catégories :

1. Les constantes physiques.

2. Les constantes chimiques.

Malheureusement ces constantes, fort nombreuses, sont d'inégale valeur et les meilleures, peut-être, sont ou trop peu connues, ou mal comprises. Leur application maladroite provoque souvent des résultats contradictoires qui justifient les reproches qu'on leur fait. Aussi comprenons-

(1) Thèse de Doctorat ès-sciences de l'Université de Nancy. 1932. 